\title{
Thermal Analyses of Polyol-Aqueous Solutions at Temperatures below $0^{\circ} \mathrm{C}$
}

\author{
Tatsuya UraJı, ${ }^{1,2}$ Hiroyuki Kohno, ${ }^{1}$ Kyoko Nakashima, ${ }^{1}$ Makoto Shimoyamada ${ }^{2}$ and Kenji WatanabE ${ }^{2}$ \\ ${ }^{1}$ Nikken Fine Chemical Co., Ltd., 24-12, Kitahama, Chita, Aichi 478, Japan \\ ${ }^{2}$ The United Graduate School of Agriculture Science, Gifu University, 1-1, Yanagido, Gifu 501-11, Japan
}

Received March 12, 1996

\begin{abstract}
The thermal properties of polyol-aqueous solutions (xylitol, sorbitol, maltitol and lactitol) in concentrations from 5\% to $30 \%\left(0.15-2.86\right.$ molal) were analyzed by differential scanning calorimetry at temperatures from $0^{\circ} \mathrm{C}$ to $-52^{\circ} \mathrm{C}$ and compared with those of their corresponding reducing sugars (xylose, glucose, maltose and lactose). The freezing point depression $(\Delta T)$ values in samples of both groups increased linearly with their concentrations (molal) and were similar to their corresponding sugars. On the other hand, the ice fraction (\%) in their samples decreased somewhat depending on the concentration. It was generally observed that the ice fraction in the polyol-aqueous solution was slightly higher than in the corresponding sugar, as estimated by plotting the ice fraction as a function of molality multiplied by the number of -OH groups.
\end{abstract}

Keywords: polyol, sorbitol, freezing point, thermal analysis, differential scanning calorimetric analysis

Generally speaking, an increase in solute concentration in an aqueous solution causes a lowering of its freezing point. Because polyols and the other sugar substitutes also have this property characteristic, they have usually been applied as a regulator of the physical property of frozen food in the food industry to provide a soft texture and a sparingly crystalline solution (Jana et al., 1994). In the previous paper (Uraji et al., $1996)$, we reported that the freezing point depression $(\Delta T)$ of some polyol-aqueous solutions in high concentration (30$60 \%$ ) was not subject to Raoult's law but successfully fitted Weast's equation on $\Delta T$ in non-ideal equations.

Many studies have been carried out on the crystallization of ice and its damage to biological tissues during the freezing of foods, because the ice formation effects the quality of frozen foods. Recently, non-frozen food as well as frozen food using polyols at low temperature below $0^{\circ} \mathrm{C}$ have become popular. Several studies to develop theoretical models of freezing point and ice crystal formation have been carried out on polyols (glycerol and mannitol), reducing sugars (glucose, fructose, lactose and maltose) and sucrose (Chen, 1986; Chen \& Nagy, 1987). An investigation on the unfrozen water content of freeze-concentrated sucrose solution was performed by a differential scanning calorimetry (DSC) method (Hatlay et al., 1991). Phase diagrams of water systems, which was one of the best methods for measurement of the ice fraction in frozen food as a function of temperature, were presented for the sucrose-water system (Young \& Jones, 1949), the D-glucosewater phase diagram (Young, 1957), and some sugars and protein solutions (Pongsawatmanit \& Miyawaki, 1993). The $\Delta T_{\mathrm{s}}$ and ice fractions of polyols and the corresponding reducing sugars were then measured.

The purpose of this study was to determine freezing points and ice formation using DSC analyses of polyol- and the corresponding sugar-aqueous solutions in a concentration range from 5 to $30 \%$ at a temperature higher than their eutectic points and below $0^{\circ} \mathrm{C}$.

\section{Materials and Methods}

Materials Xylitol (Kyowahakko Co., Ltd., Tokyo), sorbitol and lactitol-dihydrate (Nikken Chemicals Co., Ltd., Tokyo), maltitol (Towakasei Kogyo Co., Ltd., Tokyo), xylose (Xyrofin Co., Ltd., Tokyo), glucose (Yoneyama Yakuhin Kogyo Co., Ltd., Osaka), lactose-monohydrate (Kishida Kagaku Co., Ltd., Osaka), and maltose-monohydrate (Nihon Denpun Kogyo Co., Ltd., Kagoshima) were obtained from commercial sources. These polyols and saccharides were further purified by recrystallization from their concentrated aqueous solutions by cooling. The resulting products, with purities of approximately $100 \%$ shown by HPLC analysis, were used in this study.

Method of DSC analysis An instrument (SEIKO SSC/5299S II, SEIKO Instruments, Inc.) for DSC analysis was used to measure enthalpy changes in the samples. The instrument constant of the DSC was measured using standard materials with known latent heats of phase transition at a certain temperature. Distilled water, methyl benzoic acid and mercury (Wako Pure Chemical) were used for this purpose. Aqueous solutions of polyols and the corresponding sugars mentioned above were prepared at 5, 10, 20 and $30 \%$ weight per weight, respectively. About $5 \mathrm{mg}$ of each sample solution was accurately transferred into an aluminum DSC pan and the pan was sealed. Aluminum oxide was used as a reference sample. After the samples were cooled down at $1.2^{\circ} \mathrm{C} / \mathrm{min}$ from $20^{\circ} \mathrm{C}$ to $-52^{\circ} \mathrm{C}$, where all samples were recognized to be apparently frozen, the samples were heated up at a constant rate of $1.5^{\circ} \mathrm{C} / \mathrm{min}$ to $25^{\circ} \mathrm{C}$. At least two measurements were made for each sample. The enthalpy obtained from the DSC endothermic curve was used to measure the ice fraction as a function of temperature following the method of Maeda \& Koga (1981).

\section{Results and Discussion}

The freezing point could not be estimated by the exother- 


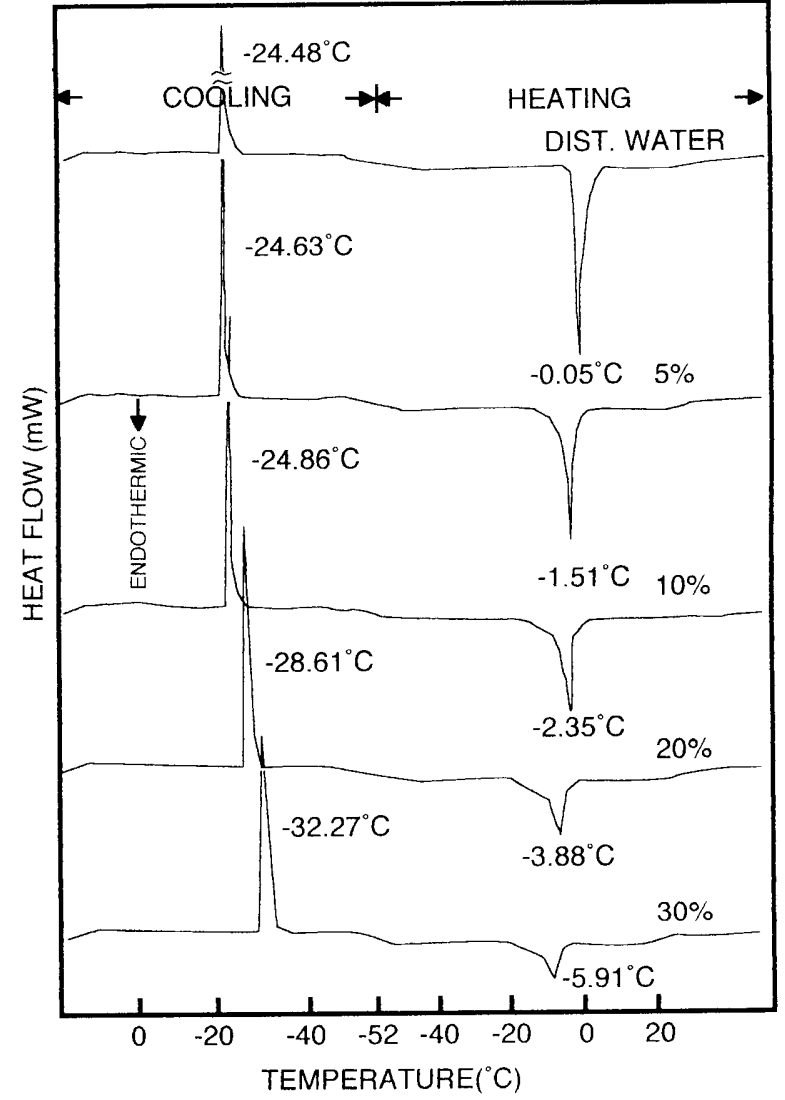

Fig. 1. DSC thermograms of sorbitol aqueous solutions from $5 \%$ to $30 \%$ mic peaks, which appeared during the period of lowering the temperature of the polyol- and the corresponding sugaraqueous solutions subjected to DSC analyses in this experiment, because the supercooling phenomenon was remarkable. For example, the peak top temperature of distilled water was $-24.48^{\circ} \mathrm{C}$, as seen in Fig. 1 .

Each peak top temperature among the endothermic peaks which appeared during the period of increasing temperature of the distilled water $\left(-0.05^{\circ} \mathrm{C}\right.$ in Fig. 1) and standard materials in DSC analyses was essentially similar to the theoretical freezing point, respectively, as in the cases estimated using the same approach for the samples described below. Therefore, the endothermic peak top temperature could be regarded as the freezing point in this experiment.

As indicated in the thermograms (Fig. 1) of the sorbitolaqueous solution, the DSC patterns were measured under a constant heating rate condition after cooling down to $-52^{\circ} \mathrm{C}$. From the thermograms, it was generally noted that the top endothermic peak temperature changed according to the concentration of the aqueous solution used. The temperature difference between the freezing point of each measured sample and distilled water was defined as the freezing point depression $(\Delta T)$.

The relationships between the $\Delta T$ of polyol- and saccharide-aqueous solutions and their molals are shown in Fig. 2. The data for each sample were regressed into Eq. (1) with a good correlation coefficient.

$$
\Delta T=A+B m
$$

$\Delta T$ : freezing point depression; $A$ : y-intercept; $B$ : slope; $m$ : molal concentration of solute.

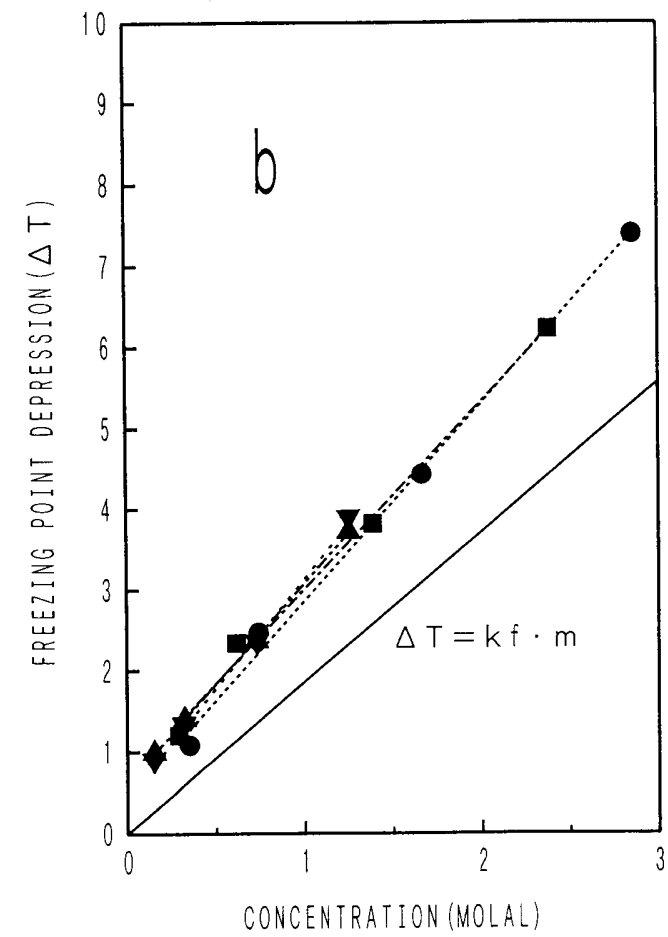

Fig. 2. Freezing point depression of polyol- and its reducing sugar-aqueous solutions as a function of concentration (molal). a, polyols: $\bigcirc$, xylitol $(A=0.76, B=$ 2.21, $r=0.9995) ; \quad$, sorbitol $(A=0.93, B=2.10, r=0.9994) ; \wedge$, maltitol $(A=0.30, B=2.80, r=0.9946) ; \vee$, lactitol $(A=0.69, B=2.41, r=0.9952) ;-$, line of theoretical $\Delta T(k f=1.855)$. b, reducing sugars: $\bullet$, xylose $(A=0.40, B=2.45, r=0.9977)$; $\mathbf{\square}$, glucose $(A=0.68, B=2.33, r=0.9970) ; \boldsymbol{\wedge}$, maltose $(A=0.59, B=2.48$, $r=0.9998)$; $\mathbf{\nabla}$, lactose $(A=0.42, B=2.71, r=0.9969) ;-$, line of theoretical $\Delta T(k f=1.855)$. 
The ideal equation is $\Delta T=B m$ in dilute solution, but Eq. (1) at high concentration solution was generally found to hold. It is clear from Figs. 2, a and b that linearities were retained in the range of 5\% to $30 \%$ in weight per weight(molal concentration from 0.153 to 2.857 ), indicating that the values of $r$ for any materials were more than 0.99 . The slopes (value $B=2.10-2.80$ ) in plots of the samples used were remarkably

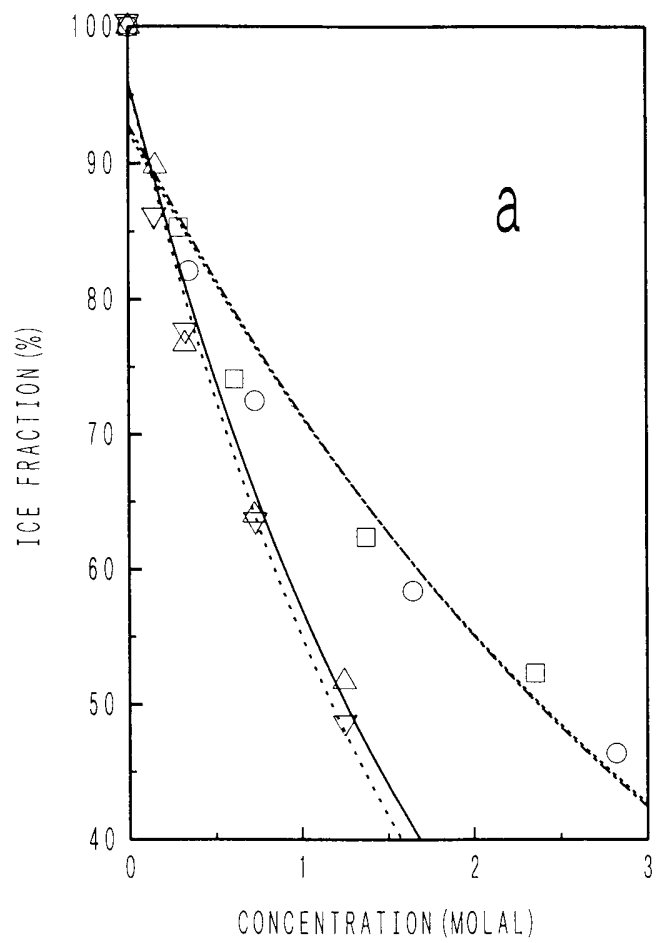

greater than that of the ideal $\Delta T(B=1.855$, Fullerton $e t$ al., 1992).

As seen in Fig. 2a, the slope for xylitol (molecular weight: 152.2) was roughly the same as that for sorbitol (molecular weight: 182.1). A slight difference in the slope between maltitol (molecular weight: 344.3) and lactitol (molecular weight: 344.3 ) could be found. Interestingly, there were no

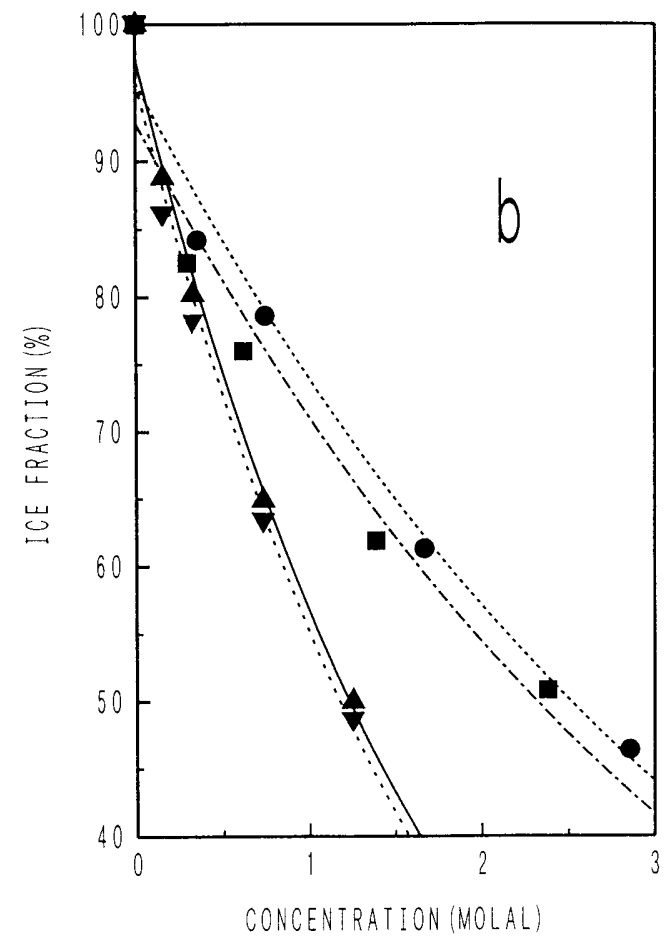

Fig. 3. Ice fraction of polyol- and its reducing sugar-aqueous solutions as a function of concentration (molal). a, polyols: $\bigcirc, \mathrm{xylitol} ; \square$, sorbitol; $\wedge$, maltitol; $\nabla$, lactitol. b, reducing sugars: $\bullet$, xylose; $\boldsymbol{\square}$, glucose; $\boldsymbol{\Delta}$, maltose; $\boldsymbol{\nabla}$, lactose.
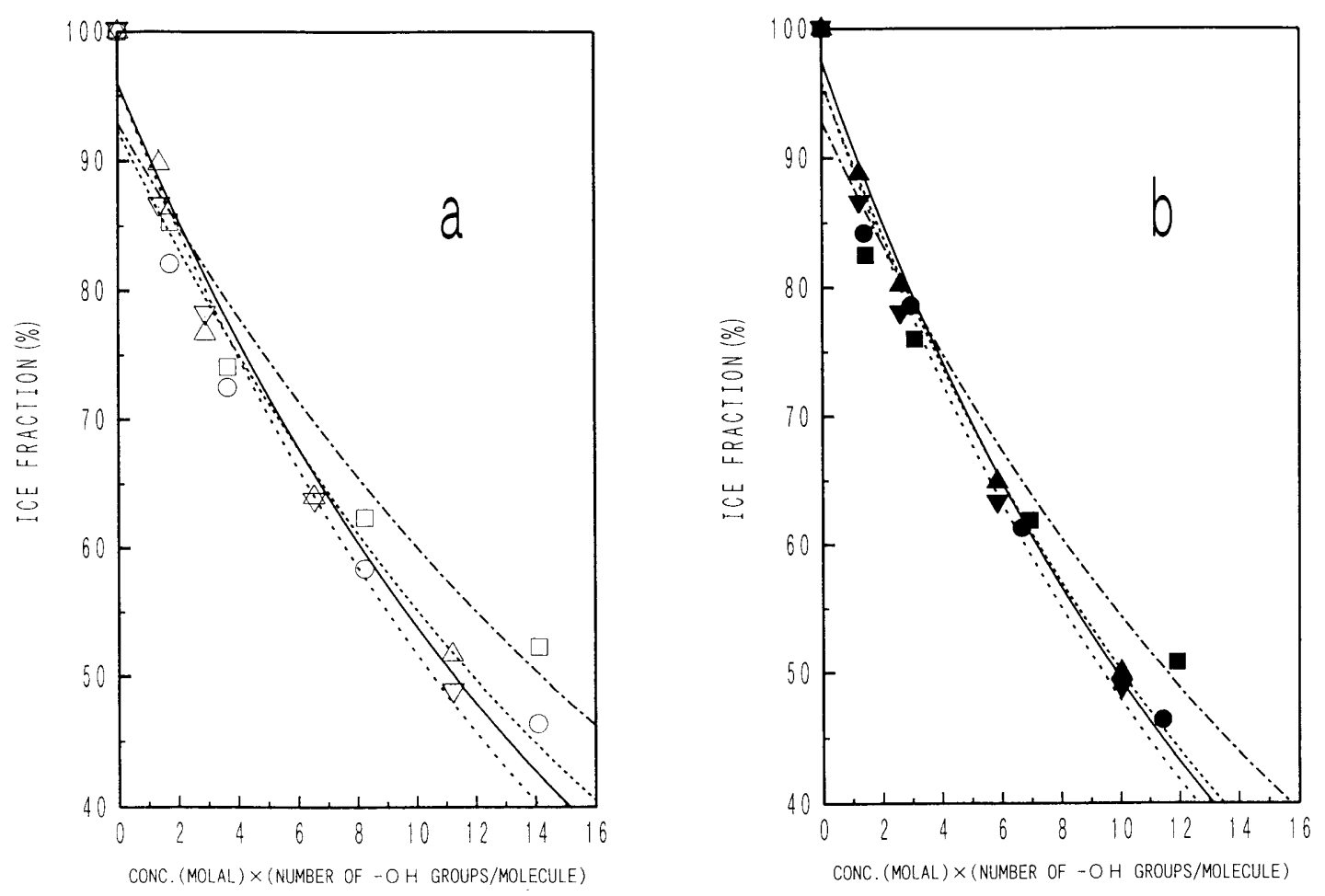

Fig. 4. Ice fraction of polyol- and its reducing sugar-aqueous solutions as a function of conc.(molal) $\times($ number of $-\mathrm{OH}$ groups $/ \mathrm{molecule})$. a, polyols: $O$, xylitol; [.]. Sorbitol; $\triangle$, maltitol; $\checkmark$, lactitol. b, reducing sugars: $\bullet$, xylose; $\mathbf{\square}$, glucose; $\boldsymbol{\Delta}$, maltose; $\mathbf{\nabla}$, lactose. 
differences in the slopes between xylitol and sorbitol, although the slopes for polyol tended to become greater with an increase in molecular weight. On the other hand, the phenomenon found in the polyols was also detected in the corresponding reducing sugars (Fig. 2b); thus there were slight differences in the slopes between xylose (molecular weight: 150.1) and glucose (molecular weight: 180.1), and the slopes for the sugars used became greater with an increase in molecular weight. The curves for each pair between the polyol and the corresponding reducing sugar were generally the same.

Next, we determined the effects of polyols and the corresponding reducing sugars on the amount of ice fraction (or freezable water), which was an important factor affecting the physical characteristics of frozen foods, by calculating the peak area of the DSC curve. The exothermic enthalpy of distilled water was $247.4 \mathrm{~mJ} / \mathrm{mg}$ at $-24.48^{\circ} \mathrm{C}$, and its endothermic enthalpy was $301.9 \mathrm{~mJ} / \mathrm{mg}$ under the conditions of this experiment. The value obtained from the latter was approximate to the known one $(332.6 \mathrm{~mJ} / \mathrm{mg}$, Fennema, 1973). The endothermic peak area in the DSC curve was also found to be more reliable than the exothermic peak area in this experiment, as described by many researchers (Boutron, 1984; Maeda \& Koga, 1981; Pongsawatmanit \& Miyawaki, 1993; Roos \& Karel, 1991; Wang \& Kolbe, 1991).

Plots between the obtained ice fraction (\%) and molality are shown in Fig. 3. The experimental endothermic enthalpy was corrected by the known endothermic enthalpy. The ice fraction gradually decreased with increasing molality in every sample. Ice fraction data determined from various samples could not be regressed into first order. The ice fraction was generally found to become smaller in the order of increasing molecular weight of the samples, as seen in the comparisons of polyol samples (Fig. 3a) and sugar samples (Fig. 3b). Their curves in both Figs. 3 , $a$ and $b$ were roughly divided into two groups according to their molecular weights, that is, the group of xylitol and sorbitol and that of maltitol and lactitol in the former, the group of xylose and glucose and that of maltose and lactose in the latter. There was no difference in the formed ice fraction between polyol and the corresponding sugar solutions in comparison of Figs. 3, a and b.

Those concentration-dependent variations could be presumed to depend not only on the number of sample molecules, but on the interaction between the sample and water molecules, and the molecular size and structure of samples. These phenomena would also appear to be caused by the higher water content in the smaller weight materials.

The influence of molecular structure on the ice formation was then estimated. Plots between ice fraction and the molal concentration multipled by the number of $-\mathrm{OH}$ groups per molecule are shown in Fig. 4, in comparison with the groups of the polyol (Fig. 4a) and the corresponding sugar (Fig. 4b) and with each other. These data gave concave curves indicating that their slopes depended on the number of $-\mathrm{OH}$ groups in the molecule. From the comparison of Figs. 4, a and b, the ice fractions of the polyols were also found to be slightly higher than those of their corresponding sugars. These differences may depend on the number of $-\mathrm{OH}$ groups per molecule, where, for example, maltitol has $9-\mathrm{OHs}$, but maltose has $8-\mathrm{OHs}$ per molecule.

The ice fraction of sorbitol and glucose solutions at a high number of $-\mathrm{OH}$ groups was slightly higher than those of the other samples, whose curves were equivalent to each other (Fig. 4). Kabayama \& Patterson (1958), Tait et al. (1972) and Uedaira et al. (1989) independently reported that there was a close correlation between hydration and the number of equatorial $-\mathrm{OH}$ groups or the number of dynamic hydration in reducing sugars. The slight difference in the curve of glucose in Fig. 4b might depend on a group such an $-\mathrm{OH}$, although that of sorbitol could not be discussed based on such a theory for hydration.

\section{References}

Boutron, P. (1984). More accurate determination of the quantity of ice crystallized at low cooling rates in the glycerol and 1,2-propanediol aqueous solutions: comparison with equilibrium. Cryobiology, 21, $183-191$.

Chen, C.S. (1986). Effective molecular weight of aqueous solutions and liquid foods calculated from the freezing point depression. $J$. Food Sci., 51, 1537-1539, 1553.

Chen, C.S. and Nagy, S. (1987). Prediction and correlation of freezing point depression of aqueous solutions. Trans. ASAE, 30, 11761180.

Fennema, O.R. (1973). Solid-liquid equilibria. Part I. Phase diagrams and freezing curves. Ch.3. In "Low-temperature Preservation of Foods and Living Matter," ed. by O.R. Fennema, W.D. Powrie and E.H. Marth. Marcell Dekker, New York, pp. 101-104.

Fullerton, G.D., Zimmerman, R.J., Cantu, III.C. and Cameron, I.L. (1992). New expressions to describe solution nonideal osmotic pressure, freezing point depression and vapor pressure. Biochem. Cell Biol., 70, 1325-1331.

Hatley, R.H.M., van den Berg, C. and Franks, F. (1991). The unfrozen water content of maximally freeze concentrated carbohydrate solutions; validity of the methods used for its determination. Cryoletters, 12, 113-124.

Jana, A.H., Joshi, N.S.S. and Sharma A.M. (1994). Sweeteners for frozen success-a review. Aust. J. Dairy Technol., 49, 98-109.

Kabayama, M.A. and Patterson, D. (1958). The thermodynamics of mutarotation of some sugars. Can. J. Chem., 36, 563-573.

Maeda, Y. and Koga, S. (1981). Thermal properties of water in Bacillus cereus spore and vegetative cells. Netsusokutei, 8, 57-61.

Pongsawatmanit, R. and Miyawaki, O. (1993). Measurement of temperature-dependent ice fraction in frozen foods. Biosci. Biotech. Biochem., 57, 1650-1654.

Roos, Y. and Karel, M. (1991). Water and molecular weight effects on glass transitions in amorphous carbohydrates and carbohydrate solutions. J. Food Sci., 56, 1676-1681.

Tait, M.J., Suggett, A., Frank, F., Ablett, S. and Quickenden, P.A. (1972). Hydration of monosaccharides: a study by dielectric and nuclear magnetic relaxation. J. Solution Chem., 1, 131.

Uedaira, H., Ikura, M. and Uedaira, H. (1989). Natural-abundance oxygen-17 magnetic relaxation in aqueous solutions of carbohydrates. Bull. Chem. Soc. Jpn., 62, 1-4.

Uraji, T., Kohno, H., Nakashima, K., Shimoyamada, M. and Watanabe, K. (1996). Freezing point depression of polyol-aqueous solutions in the high concentration range. Food Sci. Technol., Int., 2, 38-42.

Wang, D.Q. and Kolbe, E. (1991). Thermal properties of surimi analyzed using DSC. J. Food Sci., 56, 302-307.

Young, F.E. (1957). D-glucose-water phase diagram. J. Phys. Chem., 61, 616-619.

Young, F.E. and Jones, F.T. (1949). Sucrose hydrates: the sucrosewater phase diagram. J. Phys. Colloid Chem., 53, 1334-1350. 